\title{
Turbulent Flow around an Inclined Strut Mounted on a Plate and Effects of the Angle of Attack
}

\author{
by Sun-Young Kim*, Member Kazu-hiro Mori*, Member
}

\begin{abstract}
Summary
A numerical study on the turbulent flow around the strut-plate juncture is carried out for the inclined strut and for the flow with an angle of attack. Computations are made by solving three-dimensional incompressible Reynolds-averaged Navier-Stokes equation with MAC method. Turbulence is simulated by using Baldwin-Lomax model with a modified length scale for the treatment of corner flow. The flows, at the Reynolds number of $10^{5}$, show intensive unsteady behavior near the plate while the flows are steady enough off the plate. Inclination of the strut induces a lift which is generated mainly near the plate. On the other hand, the lift by an angle of attack reduces near the plate.
\end{abstract}

\section{Introduction}

Recently, juncture flows have received much attention in ship hydrodynamics due to the importance of their application to the ship appendage flow especially for high speed ships. As high speed ships require a high powering propulsion system and safe controllability, they often have more and larger appendages than conventional ships. So the estimation of the apendage resistance is critical for the prediction of the total resistance of the high speed ship. Besides, the juncture flow can affect the lift and stability/control characteristics of the appendages and the propellers located downstream by the generated horseshoe vortices from the appendages. However, there is no established method for estimating appendage resistance or appendage wake because detailed mechanism of the juncture flow is not clear yet.

To make it easy to understand and model the complicated juncture flow, many experimental and numerical studies have been carried out so far for simple strutplate juncture models. They include the full measurements of the juncture flow for a circular cylinder ${ }^{1)}$ and for the strut with a foil section ${ }^{2-6)}$ to provide a data base which is essential for the development of the computational codes. Some numerical studies ${ }^{7)-10}$ have been carried out to validate their codes comparing with those data base. However, most studies on the juncture flow have been performed for the case of a strut mounted upright on the plate with zero angle of attack.

\section{* Hiroshima University}

Received 9th July 1993

Read at the Autumn meeting 9, 10th Nov. 1993
Considering actual application of the juncture flow such as the flow around the bracket attached to the ship, more general cases are required to be studied; studies on the effect of inflow angle, inclination of the strut, curvature of the plate and so on. Nevertheless such studies are very few.

When the strut mounted on the plate inclined, the flow characteristics become quite different from the one of the upright strut. However, little attention has been paid to this problem although inclined strut is commonly used in practical application. Hoerner ${ }^{11)}$ pointed out the increase of the drag due to the inclination of the strut. However, there have been no reports on the characteristics of the flow according to the authors' knowledges. Recently, Kim and Mori ${ }^{12)}$ found out through the numerical computation, although laminar flow, the inclination of strut induces a large lift on the strut near the plate.

On the other hand, studies on the strut with an angle of attack are relatively more. Briley et al. ${ }^{13)}$ computed the juncture flow for an idealized wing with an $5^{\circ}$ angle of attack. It consists of an elliptical leading edge and a semi-infinite slab of constant thickness, so that the flow only near the leading edge is considered there. Kubendran et al. ${ }^{14)}$ visualized the juncture flow with angle of attack of $4^{\circ}$ and $8^{\circ}$ where the separation becomes severe as the angle of attack increases. Özcan and Ölcmen ${ }^{15,16)}$ measured the juncture flow with $30^{\circ}$ angle of attack at the Reynolds number of $6.6 \times 10^{5}$ and found that the flow structure is very complicated in the downstream; a single tornado vortex and $\mathrm{W}$-shaped footprint of horseshoe vortex were observed. Wood and Westphal ${ }^{17)}$ investigted the spanwise distribution of the lift of the strut mounted on the plate by the measurement of the pressure and found that the lift coefficient near the plate 
is reduced by $16 \%$ from the freestream value. Kim and Mori $^{18)}$ investigated the structure of the juncture flow with $5^{\circ}$ angle of attack at the low Reynolds number by solving the $\mathrm{N}-\mathrm{S}$ equations and showed that the lift coefficient is reduced near the plate.

In the present study, we extend the previous computations of the laminar flow ${ }^{12,18)}$ to the turbulent flow. The main objective is to investigate the characteristics of the turbulent juncture flow and forces acting on the strut for the inclined strut and the flow with an angle of attack.

The investigations are made by solving the NavierStokes equations with MAC method. To simulate high Reynolds number flows, turbulence is simulated by uing Baldwin-Lomax model with a modified length scale for corner flow, although there are still negative opinions against the appropriateness for applying an algebraic eddy-viscosity model to the complicated junture flow. The present code is validated through the comparative study ${ }^{19)}$ wih the DTRC experimental data ${ }^{2)}$ for juncture flow.

\section{Computational Method}

\section{1 Governing Equation}

The governing equations for the incompressible turbulent flow are given by the Reynolds averaged Navier -Stokes and continuity equations. By introducing Boussinesq's eddy viscosity hypothesis, they can be written in the nondimensinalized form as follows;

$$
\begin{aligned}
u_{t}+u u_{x}+v u_{y}+w u_{z}= & -p_{x}+\frac{1}{R_{n}} \nabla^{2} u+\left\{\nu_{t}\left(2 u_{x}\right)\right\}_{x} \\
& +\left\{\nu_{t}\left(u_{y}+v_{x}\right)\right\}_{y} \\
& +\left\{\nu_{t}\left(u_{z}+w_{x}\right)\right\}_{z} \\
v_{t}+u v_{x}+v v_{y}+w v_{z}= & -p_{y}+\frac{1}{R_{n}} \nabla^{2} v+\left\{\nu_{t}\left(u_{y}+v_{x}\right)\right\}_{x} \\
& +\left\{\nu_{t}\left(2 v_{y}\right)\right\}_{y}+\left\{\nu_{t}\left(v_{z}+w_{y}\right)\right\}_{z}
\end{aligned}
$$

$$
\begin{aligned}
w_{t}+u w_{x}+v w_{y}+w w_{z}= & -p_{z}+\frac{1}{\hat{R}_{n}} \nabla^{2} w \\
& +\left\{\nu_{t}\left(u_{z}+w_{x}\right)\right\}_{x} \\
& +\left\{\nu_{t}\left(v_{z}+w_{y}\right)\right\}_{y}+\left\{\nu_{t}\left(2 w_{z}\right)\right\}_{z}
\end{aligned}
$$$$
u_{x}+v_{y}+w_{z}=0
$$

where subscripts represent partial differentiation with respect to the referred variables except $\nu_{t}$ and $(u, v, w)$ and $p$ are the velocity components in $(x, y, z)$-direction of the cartesian coordinates and the static pressure respectively. $\mathrm{t}$ is the time and $\nu_{t}$ represents the eddy viscosity.

All the variables are nondimensionalized by the chord length of the strut $L$, the uniform flow velocity $U_{0}$, and the density of water $\rho, R_{n}$ is the Reynolds number based on $U_{0}$ and $L$.

A body-fitted coordinate system is adopted and transformation is given by

$$
\xi=\xi(x, y, z), \eta=\eta(x, y, z), \quad \zeta=\zeta(x, y, z)
$$

With this relations, the following transformed governing equations are obtained.

$$
u_{t}+U u_{\xi}+V u_{\eta}+W u_{\xi}=-\left(\xi_{x} p_{\xi}+\eta_{x} p_{\eta}+\zeta_{x} p_{\xi}\right)
$$

$$
\begin{aligned}
&+\frac{1}{R_{n}} \nabla^{2} u+R_{x} \\
& v_{t}+U v_{\xi}+V v_{\eta}+W v_{\xi}=-\left(\xi_{y} p_{\xi}+\eta_{y} p_{\eta}+\zeta_{y} p_{\xi}\right) \\
&+\frac{1}{R_{n}} \nabla^{2} v+R_{y} \\
& w_{t}+U w_{\xi}+V w_{\eta}+W w_{\xi}=-\left(\xi_{z} p_{\xi}+\eta_{z} p_{\eta}+\zeta_{z} p_{\xi}\right) \\
&+\frac{1}{R_{n}} \nabla^{2} w+R_{z} \\
& \xi_{x} u_{\xi}+\eta_{x} u_{\eta}+\zeta_{x} u_{\xi}+\xi_{y} v_{\xi}+\eta_{y} v_{\eta}+\zeta_{y} v_{\xi}+\xi_{z} w_{\xi} \\
&+\eta_{z} w_{\eta}+\zeta_{z} w_{\xi}=0
\end{aligned}
$$

where $U, V$ and $W$ are the contravariant velocity components defined as

$$
\begin{aligned}
& U=\xi_{x} u+\xi_{y} v+\xi_{z} w \\
& V=\eta_{x} u+\eta_{y} v+\eta_{z} w \\
& W=\zeta_{x} u+\zeta_{y} v+\zeta_{z} w
\end{aligned}
$$

Laplacian $\nabla^{2}$ can be expressed in the body fitted coordinates in the following form;

$$
\begin{aligned}
\nabla^{2} q= & \hat{a} q_{t \xi}+\hat{b} q_{\eta \eta}+\hat{c} q_{\xi \xi}+2 \hat{d} q_{\xi \eta}+2 \hat{e} q_{\eta \xi} \\
& +2 \hat{f} q_{k \xi}+\hat{g} q_{\xi}+\hat{h} q_{\eta}+\hat{\imath} q_{\xi}
\end{aligned}
$$

$R_{x}, R_{y}$ and $R_{z}$ represent the Reynolds stress terms transformed from the last three terms of RHS of Eq. (1).

\section{2 Numerical Scheme and Boundary Condition}

The computations are made by MAC method where Poisson equation for the pressure is derived by taking a divergence of the momentum equation and satisfying the continuity equation. The Poisson equation for the pressure is solved iteratively by using the relaxation method and the velocities are updated from the momen. tum equation.

The finite difference equations are derived on a regular grid system. So all the variables are defined on the grid nodes. For the spatial differencing scheme, 2nd order central difference is used while the upwind scheme for the convective term. The Euler explicit scheme is used for the time marching procedure. The computation starts from the still state and the flow is accelerated up to a given constant velocity for the numerical stability.

A C-type grid is adopted for the computation. The coordinate system and grid topology are shown in Fig. 1. In the physical domain, a space-fixed cartesian coor-

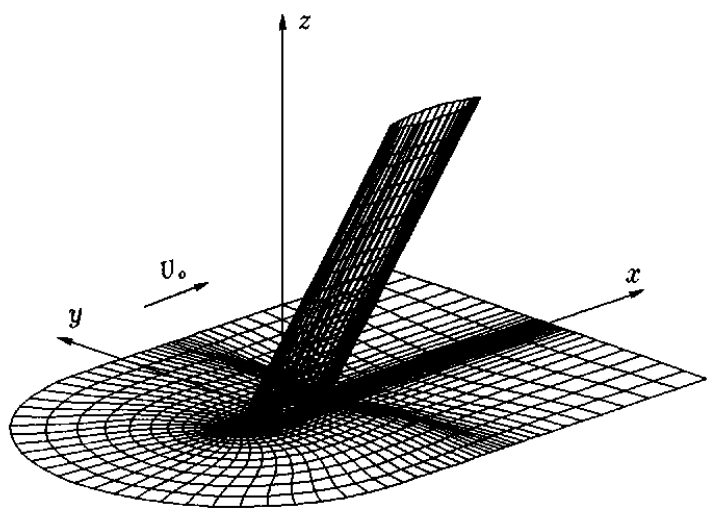

Fig. 1 Grid and Coordinate System 
dinate system is usd whose origin is located at the center of the strut on the plate and $x^{-}, y$ - and $z$-axes are in the uniform flow, lateral and vertical direction respectively. In the transformed body-fitted coordinate system, $\xi$ - and $\eta$-axes are the girthwise of the strut and the normal to the surface of strut and $\zeta$-axis coincides with $z$-axis. Therefore the surface of strut lies in the $\eta$ constant plane and the plate lies on the $\zeta$-constant plane.

On the strut and the plate, no-slip condition is used for the velocity and Neumann condition is obtained from the momentum equation for pressure. Outer boundaries are taken on the plate including the inflow and the lateral boundaries where a 1/7-th boundary layer profile with constant pressure is prescribed while the $v$-component of velocity is obtained from the momentum equation. The inflow profile is determined by specifying the position of the leading edge of the plate. The height of the strut is assumed to be infinite and the zero gradient conditions are used for all the variables. On the downstream boundary, the velocities are linearly extrapolated in the streamwise direction by assuming that the diffusion in the streamwise direction is negligible but zero gradient condition is used for the pressure.

\subsection{Turbulence Model}

The turbulence model for the flow around the juncture is complicated and not well developed yet. In this study, the turbulence transport is described by using an algebraic eddy-viscosity model proposed by Baldwin \& Lomax ${ }^{20)}$ which has been used widely in computational fluid dynamics for its simplicity. Modified length scale ${ }^{21)}$ is incorporated into a Baldwin-Lomax model to treat the corner flow where the turbulence is influenced by both plate and strut.

The procedure of applying Baldwin-Lomax model for the corner flow is as follows. As sketched in Fig. 2, in each $(\mathrm{J}-\mathrm{K})$ sectional plane, the computational domain is a corner formed by two walls. The local coordinates $\left(n_{y}, n_{z}\right)$ will be used for the discussion which has an orign on the corner. The eddy viscosity $\nu_{t}$ is computed in the inner and outer regions separately. In the inner region, the eddy viscosity is given by the Prandtl-van Driest formulation

$$
\left(\nu_{t}\right)_{\text {inner }}=(\kappa D l)^{2}|\omega|
$$

were $\kappa=0.4$ is von Karman's constant and $|\omega|$ is the magnitude of the vorticity. The definition of $l$ is critical for the evaluation of the eddy viscosity. Bleev's mixing length $^{21)}$ for the open corner formed by the normal intersection of two planes is used for the present study.

$$
l=\frac{2 n_{y} n_{z}}{n_{y}+n_{z}+\left(n_{y}^{2}+n_{z}^{2}\right)^{1 / 2}}
$$

When the open corner has an arbitrary intersection angle $\phi$, more general formula ${ }^{19)}$ for characteristic length $l$ is given by

$$
l=\frac{2 r n_{y} n_{z}}{r\left(n_{y}+n_{z}\right)+\bar{y}\left(n_{y}+n_{z} \cos \phi\right)+n_{z}^{2} \sin \phi}
$$

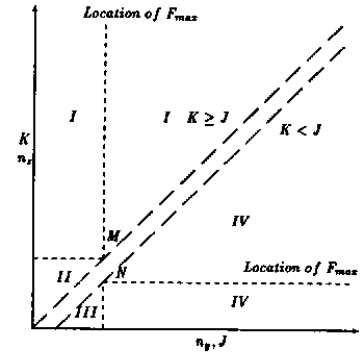

(a)

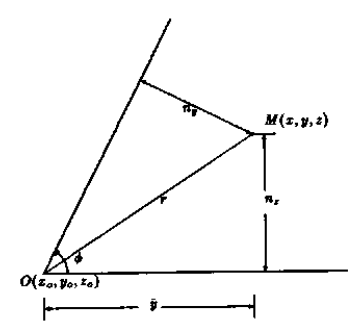

(b)
Fig. 2 Definition for the Corner Flows

where the definition of notaion is shown in Fig. 2(b). The van Driest damping factor $D$ is given by and

$$
D=1-\exp \left(-l^{+} / 26\right)
$$

$$
l^{+}=\frac{u_{\tau} l}{\nu}=\frac{l}{\nu} \sqrt{\frac{\tau_{w}}{\rho}}
$$

where $\tau_{w}$ is the wall shear stress.

In the outer region,

$$
\left(\nu_{t}\right)_{\text {outer }}=k C_{c \rho} F_{\text {wake }} F_{\text {kleb }}
$$

where

$$
F_{\text {wake }}=\text { the smaller of }\left\{\begin{array}{l}
l_{\max } F_{\max } \\
0.25 l_{\max } U_{D I F}^{2} / F_{\max }
\end{array}\right.
$$

The quantity $U_{D I F}$ is the difference between the maximum and the minimum resultant velocity in the profile.

$$
U_{D I F}=\left(\sqrt{\left.u^{2}+v^{2}+w^{2}\right)_{\mathrm{MAx}}}-\left(\sqrt{u^{2}+v^{2}+w^{2}}\right)_{\mathrm{MIN}}\right.
$$

In the above formulation, $k=0.0168$ is the Clauser's constant and the qauntity $F_{\max }$ is the maximum value of $F(l)=l|\omega| D$, and $l_{\max }$ is the value of $l$ at which $F_{\max }$ occurs. The Klebanoff intermittency factor $F_{\text {kleb }}$ is given by

$$
F_{\text {kleb }}=\left[1+5.5\left(\frac{C_{\text {kleb }} l}{l_{\text {max }}}\right)^{6}\right]^{-1}
$$

In the outer formulation, the constants $C_{c p}$ and $C_{\mathrm{kleb}}$ depend on the condition of flow ${ }^{22}$. In this study, however, $C_{c p}=1.6$ and $C_{k 1 e b}=0.3$ are used, which were recommended originally by Baldwin and Lomax ${ }^{20)}$. There are some ambiguity in finding $F_{\max }$ because sev. eral peaks are present in the profile of $F$ for the complex flow as pointed out by Visbal and Knight ${ }^{12)}$. The outermost peak is chosen as $F_{\max }$ among several peaks to avoid the abrupt change of the computed length scale in the streamwise direction. However, if the chosen peak is smaller than the $20 \%$ of the maximum value of $F$ in the profile, it is discarded to avoid finding the peak in the outside of the boundary layer.

The plane is divided into four regions I, II, III and IV as shown in Fig. 2. In regions I and II, $D$ is evaluated at the wall $n_{y}=0$ for region I and in regions III and IV, it is evaluated at the wall $n_{z}=0$. The search for $F_{\max }$ proceeds outward from the wall either from $n_{y}=0$ for the region I or from $n_{z}=0$ for the region IV. The values 
of $F_{\max }$ in the regions II and III are constant, equal to the value of $F_{\max }$ at $\mathrm{M}$ and $\mathrm{N}$ respectively.

Similar treatment is also applied to the wake region by assuming the slip wall on the center wake plane.

\section{Results and Discussion}

Computations are made for two cases which are the flow with an angle of attack of $-5^{\circ}$ and for the inclined strut by $30^{\circ}$. Both are at the Reynolds number of $10^{5}$. The angle of attack and inclination angle are denoted here by $\alpha$ and $\phi$ respectively. The flow is assumed to be fully turbulent so that the transition on the strut is not considered.

The strut has a spanwise constant section of NACA $16-018$ with a thickness of $18 \%$ of the chord length $L$. The leading edge of the plate is $10.5 \mathrm{~L}$ apart from the center of the strut but infinite in lateral and downstream directions. The oncoming boundary layer thickness is $0.234 L$ at the leading edge of the strut.

The computing conditions and grids for the two cases studied in this paper are summarized in Table 1.

\subsection{Inclined Strut}

The most prominent feature of the juncture flow for the inclined strut is that the flow becomes unsymmetric close to the plate due to the interaction of the flow with the plate. This is clearly shown in Fig. 3 where the velocity vectors and the pressure close to the plate are shown. Here, the side of the strut is expressed in terms of the angle between ths strut and the plate such as $60^{\circ}$-side and $120^{\circ}$-side. The flow near the leading edge is similar to the flow with an angle of attack (see Figs. 14 and 15) ; both the nodal point of attachment and the stagnation point are shifted to the $60^{\circ}$-side. The unsymmetric pressure distribution suggests an existence of a lateral (lift) force. Such characteristics of the flow are similar to those of the laminar flow ${ }^{12)}$ but the flow near the trailing edge is much more complicated.

Fig. 4 shows the time history of the forces acting on the strut where $C_{d f}, C_{d p}, C_{l s}$ and $C_{l p}$ represent the coefficients of the frictional drag, pressure drag and the frictional and pressure lift respectively. All the coefficients are nondimensionalized by $0.5 \rho U_{0}^{2} L^{2}$. The forces are oscillating with a period of about 3.0 nondimensional time. The Strouhal number based on the maximum thickness of the strut is 0.07 . The sectional force coefficients at three different $z$ are plotted versus time in Fig. 5 where the sectional force is the force acting on the strip of the strut with unit height of span. They show the same period of oscillation with the same

Table 1 Computing Conditions

\begin{tabular}{|l|c|c|}
\hline Case & $5^{\circ}$ Angle of Attack & $30^{\circ}$ Inclined Strut \\
\hline Computing & $-2.3 \leq x \leq 3.0$ & $-2.3 \leq x \leq 3.0$ \\
Domain & $-1.8 \leq y \leq 1.8$ & $-1.8 \leq y \leq 1.8$ \\
& $0.0 \leq z \leq 1.0$ & $0.0 \leq z \leq 3.0$ \\
\hline No. of Grids & $79 \times 31 \times 27$ & $79 \times 31 \times 35$ \\
\hline Minimum Grid Spacing & 0.0002 & 0.0002 \\
\hline Time Increment & 0.0002 & 0.0002 \\
\hline
\end{tabular}
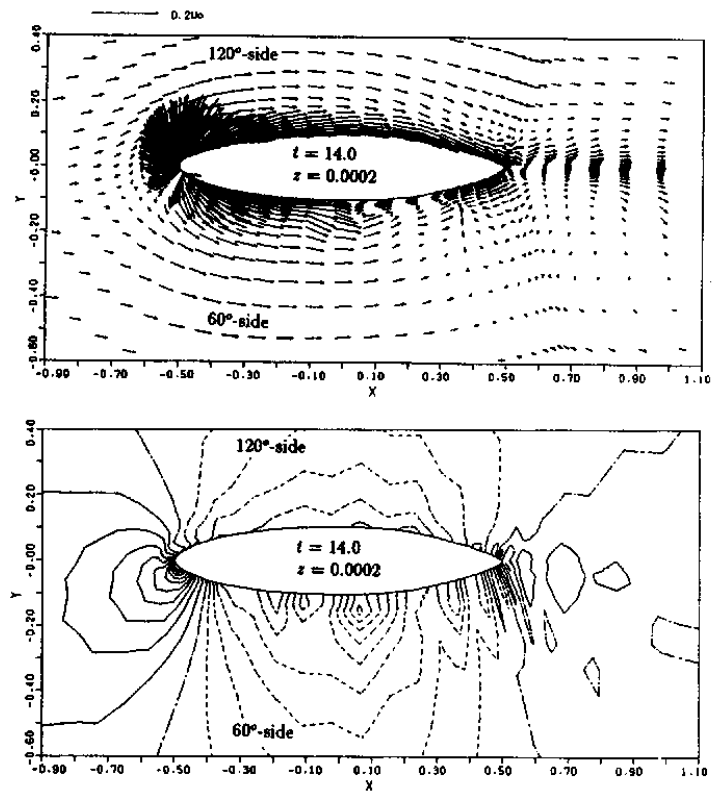

Fig. 3 Velocity Vectors and Pressure on the Plane of $z=0.0002: \phi=30^{\circ}, t=14.0$

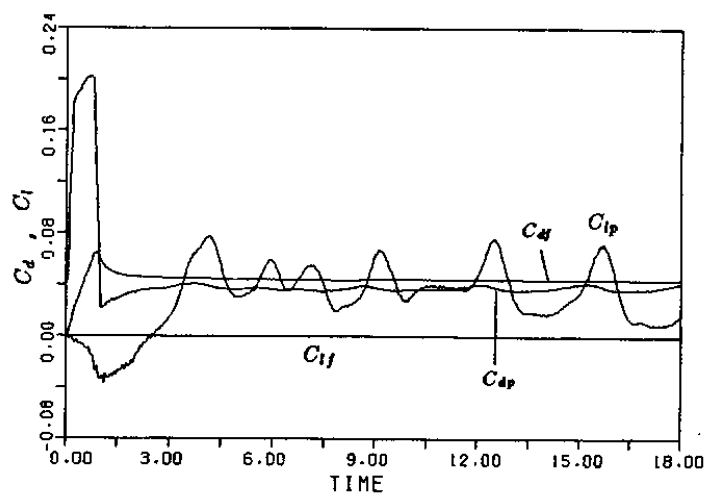

Fig. 4 Time Histories of the Forces Acting on the Strut : $\phi=30^{\circ}$

phase regardless of the height while the amplitude rapidly decreases as $z$ increases so that the flow is almost steady aparting from the plate. The result makes us eliminate the Karman vortex shedding as a possible source of the oscillation, for it would decrease near the plate due to the viscous damping contrary to the results. Thus the oscillatory forces can be supposed due to the unsteadiness of the flow near the plate.

The above guess is supported by the flows shown in Fig. 6 which are much changing with the height. The stagnation point is shifted to the $60^{\circ}$-side near the plate but it moves to the leading edge to be symmetry as $z$ increases. The flow separated from the plate around 

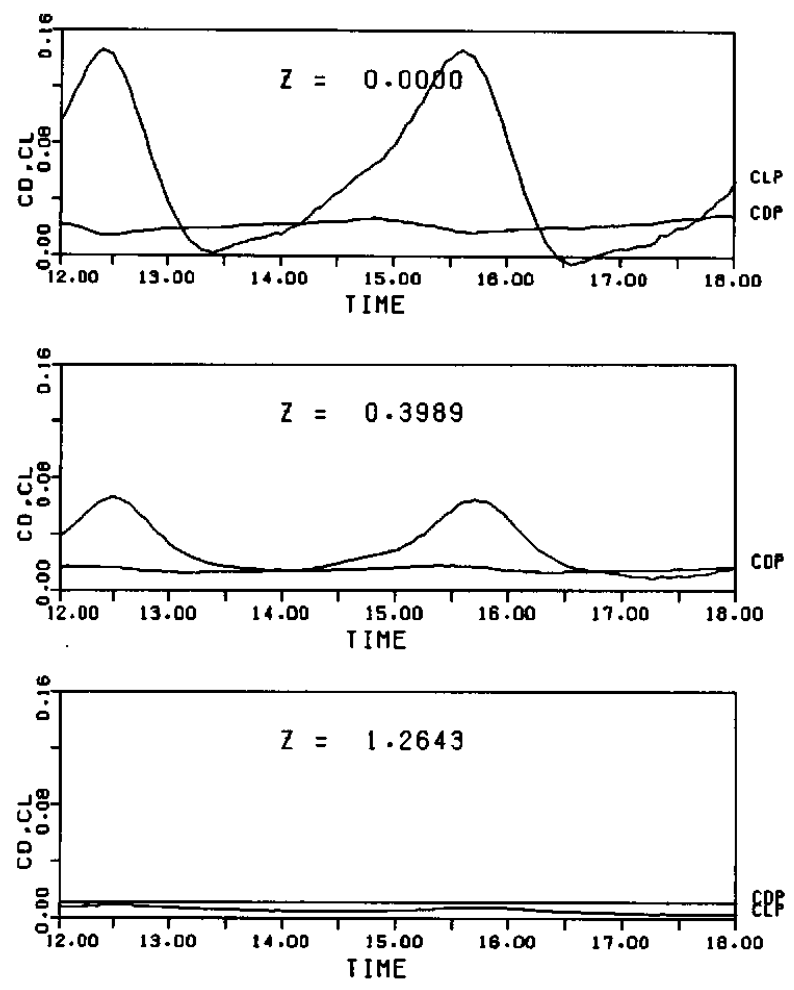

Fig. 5 Time Histories of the Sectional Forces of the Strut at Three Different Heights : $\phi=30^{\circ}$

$x=0.35$ and $y=-0.2$ (Fig. 6(a)) makes a large scale of uprising vortical flow (Fig. 6(b)) while the flow is almost two-dimensional on the plane of $z=0.4$.

Fig. 7 shows the cross-flow velocity at several planes of $x=$ constant. It shows quite different flows on both sides. At the plane of $x=0.4$, close to the trailing edge, an uprising separated flow from the plate is clearly seen on the $60^{\circ}$-side which has shown in Fig. 6 , too. In the wake, $x=0.7$, there is only one dominant trailing vortex with an anti-clockwise direction on the $60^{\circ}$-side. Attachment of the flow can be also seen there.

Fig. 8 shows the instantaneous velocity vectors at three different times on the closest plane to the plate. Except around the leading edge, the flows are much changing especially on the $60^{\circ}$-side. There are several singular points such as spiral nodal points of separation and nodal points of attachment whose patterns are changing with time. This means that the patterns of the separation and the attachment of the flow are changing with time near the plate.

Fig. 9 shows schematically the evolution of the flow topology on the plate correspondig to the flows shown in Figs. 4, 7 and 8 . They satisfy the following topology rule $^{23)}$

$$
\left(\Sigma_{N}+1 / 2 \Sigma_{N^{\prime}}\right)-\left(\Sigma_{s}+1 / 2 \Sigma_{S^{\prime}}\right)=-1
$$
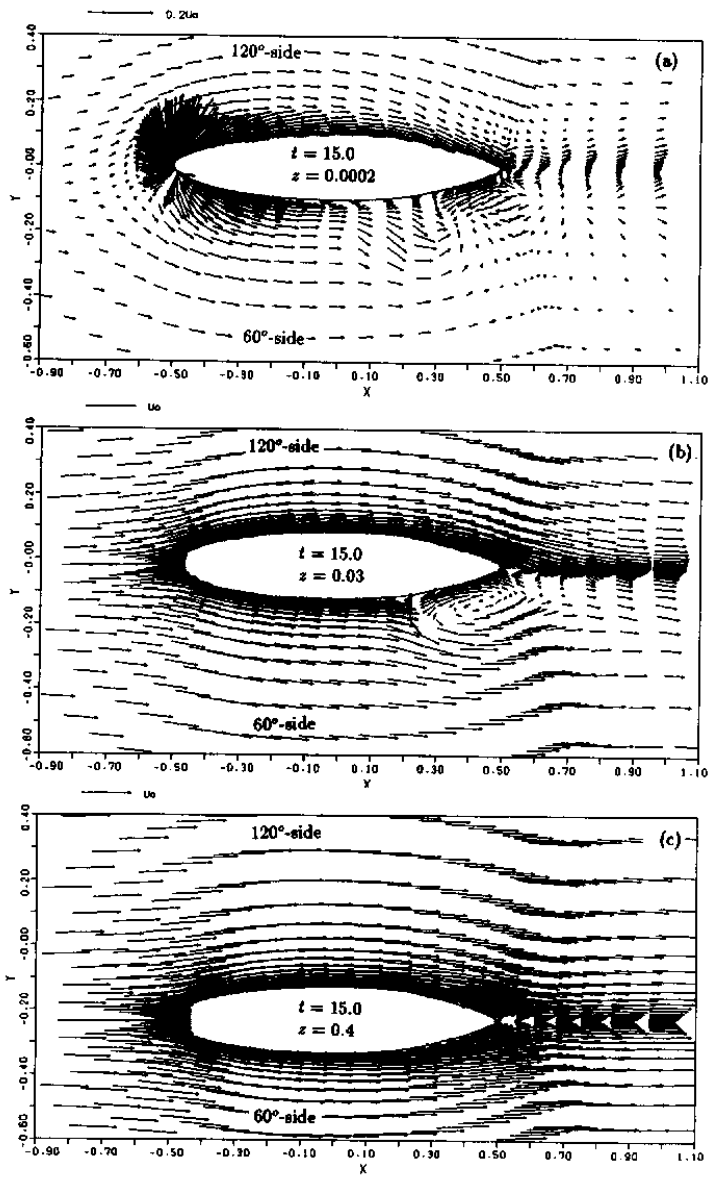

Fig. 6 Volocity Vectors on the Plane of $z=$ constant : $\phi=30^{\circ}, t=15.0$

where $\Sigma_{N}, \Sigma_{N^{\prime}}, \Sigma_{s}$ and $\Sigma_{s^{\prime}}$ represent the number of nodes $(N)$, half-nodes $\left(N^{\prime}\right)$, saddles $(S)$ and half-sad. dles $\left(S^{\prime}\right)$ respectively. The flow at $t=14.0$ shows the simplest topology among all shown in Fig. 9. There are three saddle points and four half-saddle points. The nodal points, $N_{1}$ and $N_{4}$ are the nodal points of attachment while $N_{2}$ and $N_{3}$ are the focal nodal points of separation. That is to say, the flow attaches to the plate and spreads over the plate at $N_{1}$ and $N_{3}$ while the flow separates at $N_{2}$ and $N_{3}$ to become an uprising spiral vortical flow. A new nodal point of separation $\left(N_{5}\right)$ appears at $t=15.0$ but it changes its type from a regular nodal point to a focal nodal point at $t=15.5$. At $t=16.0$, that type of focal nodal point disappears while a new nodal point of attachment $\left(N_{5}\right)$ appears with a focal nodal point of separation $\left(N_{6}\right)$ which disappears at $t=$ 16.5 .

The entire process is repeated periodically. From the above process, we can understand that the main mechanism of the unsteady flow is due to the periodic separa- 

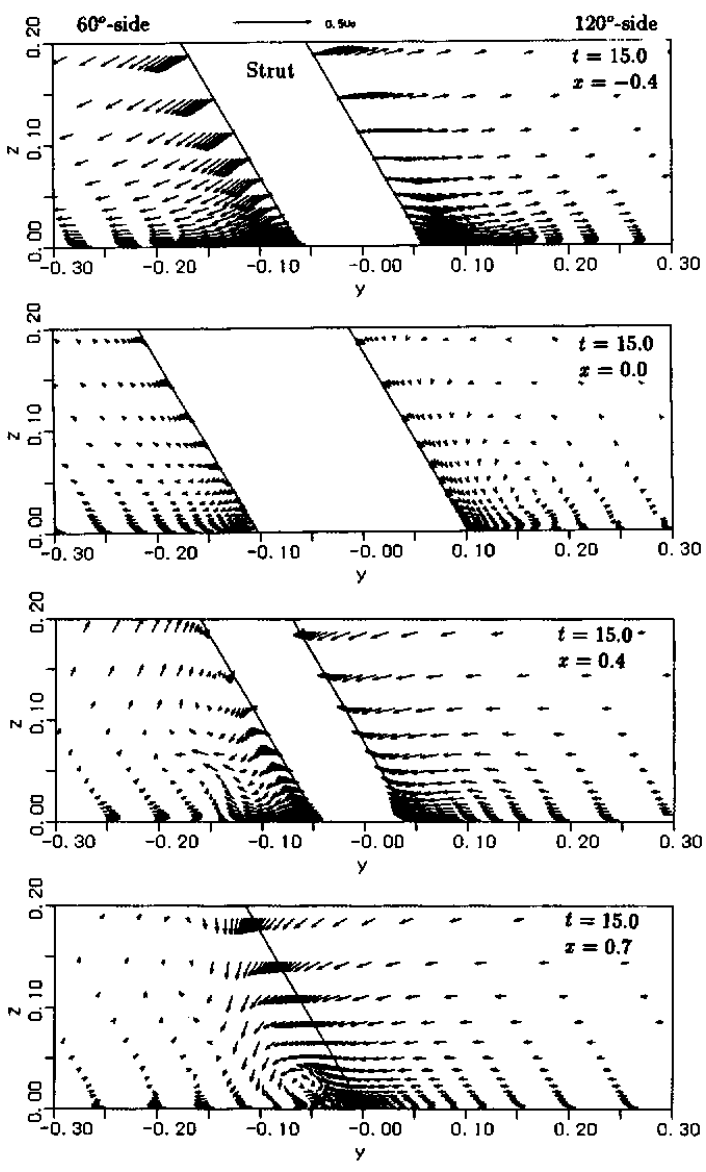

Fig. 7 Cross Flow Velocity Vectors at the Plane of $x=$ constant : $\phi=30^{\circ}, t=15.0$

tion of the flow at $N_{5}$ in Fig. $9(\mathrm{c})$ and at $N_{6}$ in Fig. 9 (d).

Fig. 10 shows the pressure distributions along the strut on $z=0$. at three different times. The pressure does not vary with time near the leading edge but it changes drastically on the aft part of the strut especially on the $60^{\circ}$-side due to the influence of the periodic separation of the flow from the plate which is the main cause for the oscillation of the lift and drag for strut shown in Fig. 4.

In Fig. 11, the computed pressures on the strut are compared with the experimental data ${ }^{24}$ which were measured in the circulating water channel at the Reynolds number of $1.45 \times 10^{5}$. The computed pressures are the averaged data from $t=12.0$ to $t=18.0$ while the measured data are the average for 12 seconds (15 nondimensional time). Although exact quantitative comparison cannot be made because of the difference of the oncoming flow in the boundary layer on the plate, they agree qualitatively well with each other; the difference of the pressure between the $60^{\circ}$-side and $120^{\circ}-$ side is large near the plate but it decreases as $z$
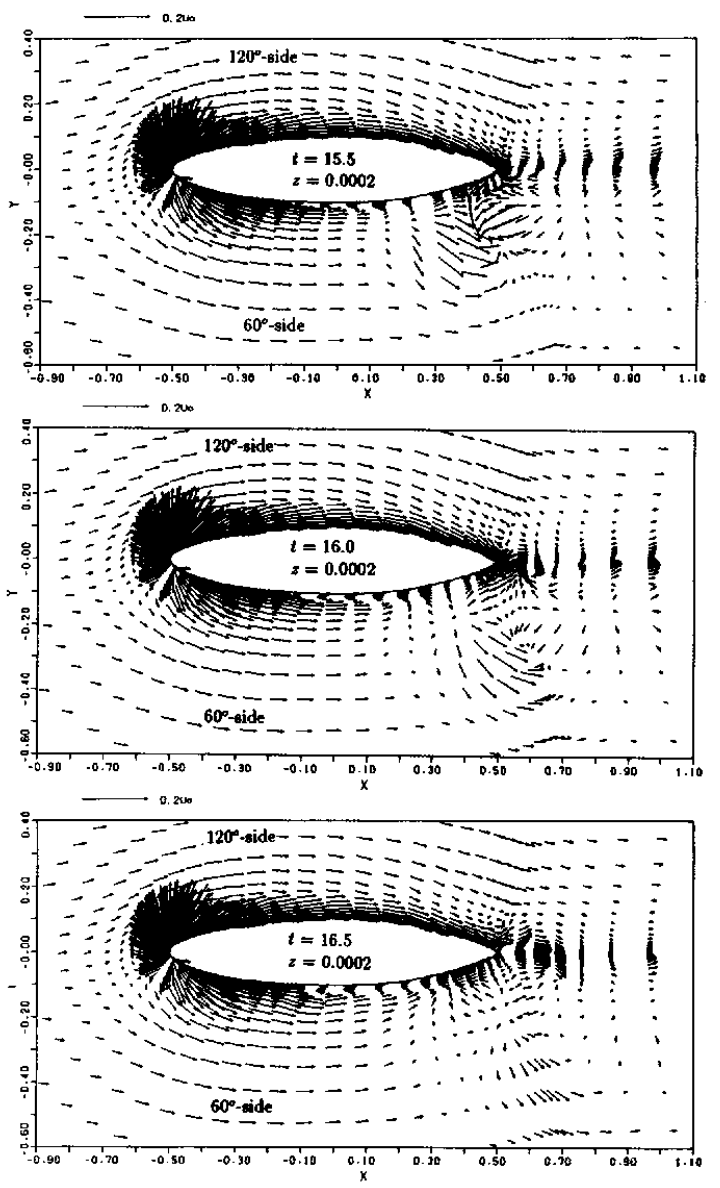

Fig. 8 Velocity Vectors on the Plane of $z=0.0002$ at Different Times : $\phi=30^{\circ}$

increases. In the middle and aft part of the strut, the computation underpredicts the pressure near the plate but the difference becomes smaller as $z$ increases. In the computed results, there are large differences of the pres. sure between the $60^{\circ}$-side and $120^{\circ}$-side around the aftbody of the strut but they are not seen in the experimental results. The difference between the computed and the experimental pressure in the aft-body of the strut cannot be explained reasonably at present although the blockage effect of the channel may be considered as a possible source for the difference.

Fig. 12 shows the spanwise distribution of the sectional drag and lift coefficients of the strut; $C_{d f 2 D}$, $C_{d p 2 D}$ and $C_{C D 2 D}$ here represent the sectional coefficients of the frictional drag, pressure drag and pressure lift respectively. All the coefficients are nondimensionalized by $0.5 \rho U_{0}^{2} L$. The computed force coefficients are obtained by averaging the data between $t=12.0$ and $t=$ 18.0. The frictional drag coefficient increases as $z$ increases and it becomes constant beyond the boundary layer thickness. The pressure drag is greater near the 


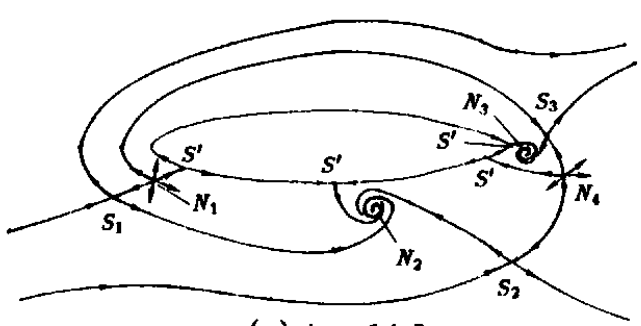

(a) $t=14.0$

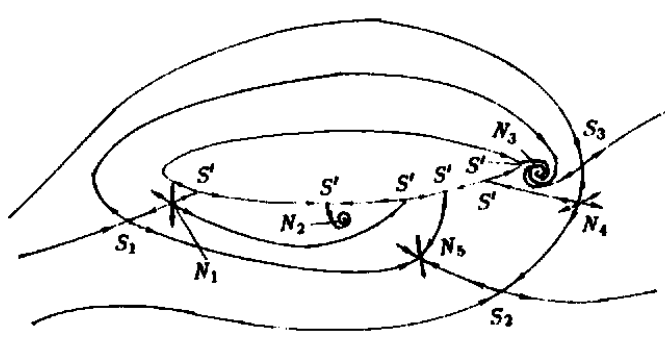

(b) $t=15.0$

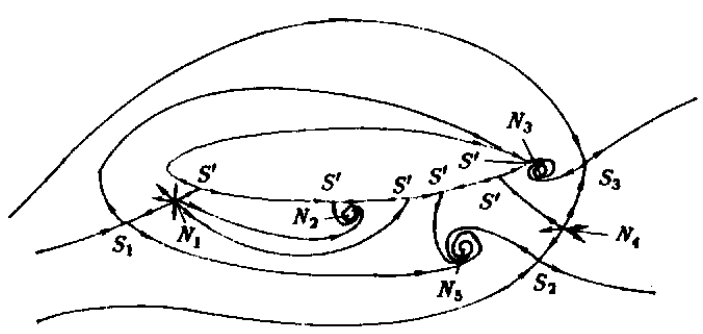

(c) $t=15.5$

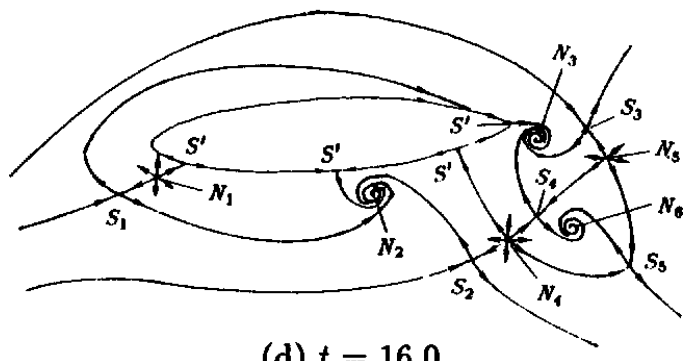

(d) $t=16.0$

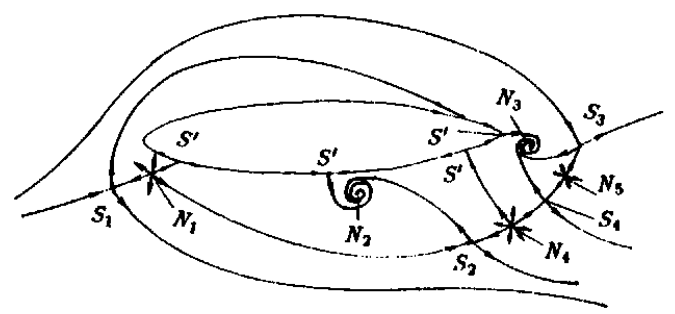

(e) $t=16.5$

Fig. 9 Evolution of the Flow Topology on the Plate: $\phi=30^{\circ}$
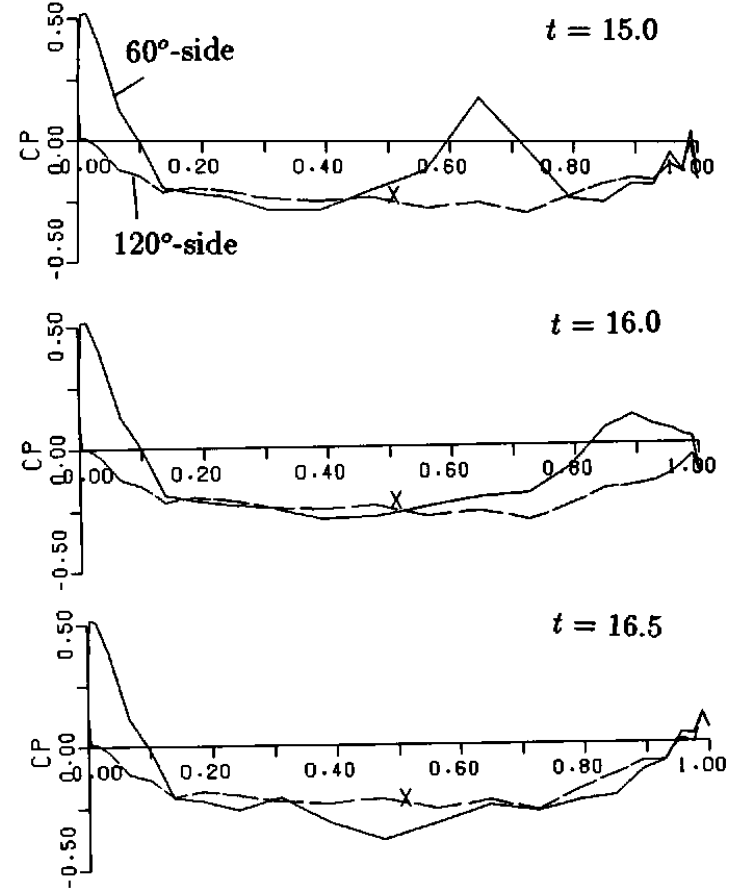

Fig. 10 Pressure Distribution along the Strut at Different Times; $\phi=30^{\circ}, z=0.0002$

plate than that beyond the boundary layer while the pressure drag was smaller near the plate in the laminar flow ${ }^{12)}$. The lift near the plate is striking and it is generated even beyond the oncoming boundary layer thickness.

Fig. 13 shows the comparison of the forces acting on the strut between computation and measured data ${ }^{25)}$. The computed forces are obtaind by integrating sectional forces up to $z=0.866$ which corresponds to the height of the strut in experiments. The force coefficients are nondimensionalized by $0.5 \rho U_{0}^{2} L^{2}$. The experimental data shows considerable lift all over the measured Reyolds numbr range. The computed forces show an agreement with experimental data. However, it should be noted that there are some differences in condition between the computation and the experiment such as oncoming boundary layer flows and finite span of the strut. Anyway it may be worthwhile to point out again that a lift force is produced by the inclination of the strut whose magnitude is about $20 \%$ of that with an attack angle of $5^{\circ}$ (see Fig. 20).

\subsection{Flow with Angle of Attack}

Fig. 14 and 15 show the comparison of the velocity and the pressure between on the plane closest to the plate and the plane above the boundary layer. Here we can see also significant effects of the plate on the flow around the strut. The flow close to the plate is very similar to that about the inclined strut near the leading 

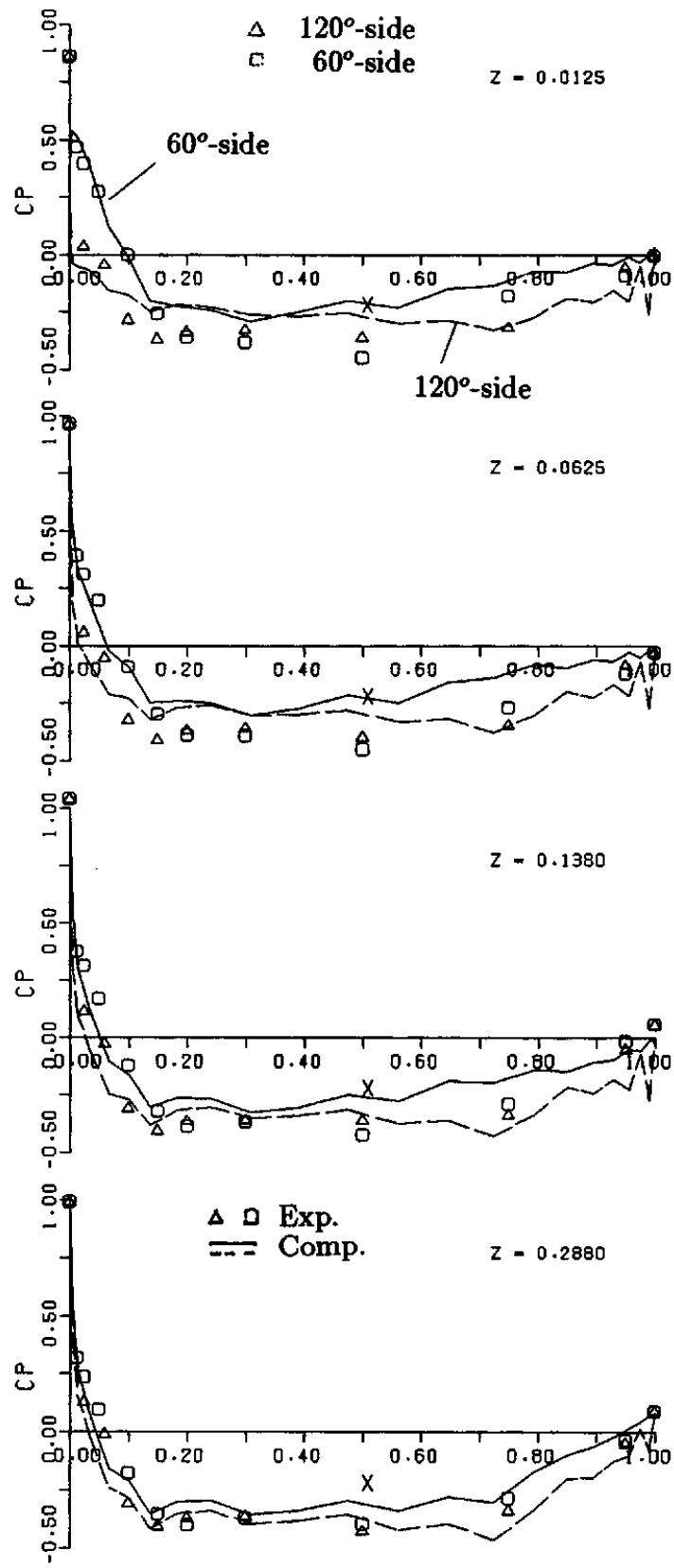

Fig. 11 Comparison of the Pressure Distribution along the Strut between Computations and Experi. mental Data[24] : $\phi=30^{\circ}$

edge but it is quite different near the trailing edge (see Fig. 3) ; the separation occurs earlier on the opposite side to the side on wihch the nodal point of attachment or the stagnation point appears. It can be seen that there are many singular points around the trailing edge which make the flow complicated and three-dimensional. On the plane above the boundary layer, their

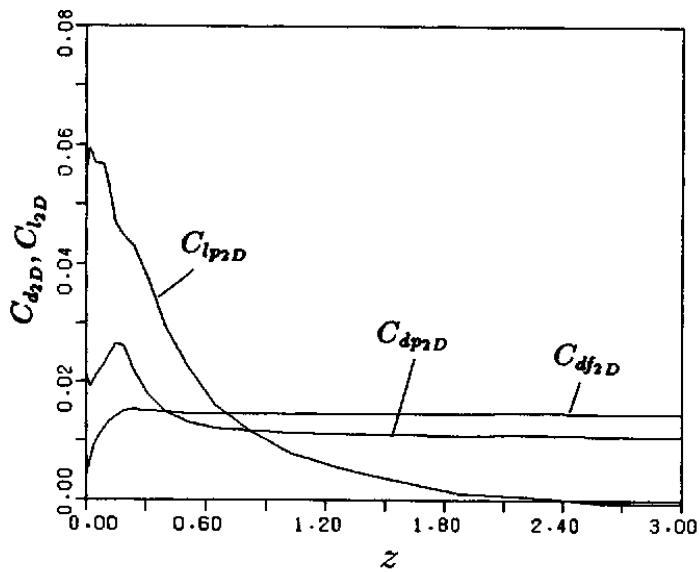

Fig. 12 Spanwise Distribution of the Sectional Drag and Lift Coefficients of the Strut : $\phi=30^{\circ}$

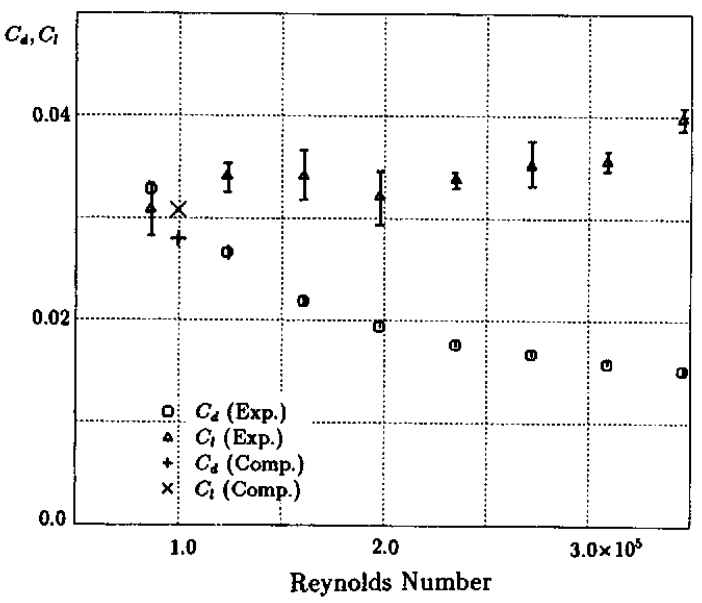

Fig. 13 Comparison of the Forces acting on the Inclined Strut between Computation and Mea. sured Data[25]

effects disappear so that the separation is much delayed very close to the trailing edge and the flow is twodimensional without any effects of the plate. Near the plate, the stagnation pressure becomes lower due to the velocity defect of the oncoming boundary laryer and the pressure does not so much drops on the suction side. This is because the acceleration of flow is hindered by the boundary layer on the plate as seen in Fig. 14.

Fig. 16 shows the time history of the forces acting on the strut. As same with the flow for inclined strut, the flow is unsteady, although the flow has not reached the periodic state even at $t=25$.

Fig. 17 shows the pressure distributions along the strut at two typical times which correspond to the time at which the hump $(t=20.46)$ and hollow $(t=21.96)$ of 

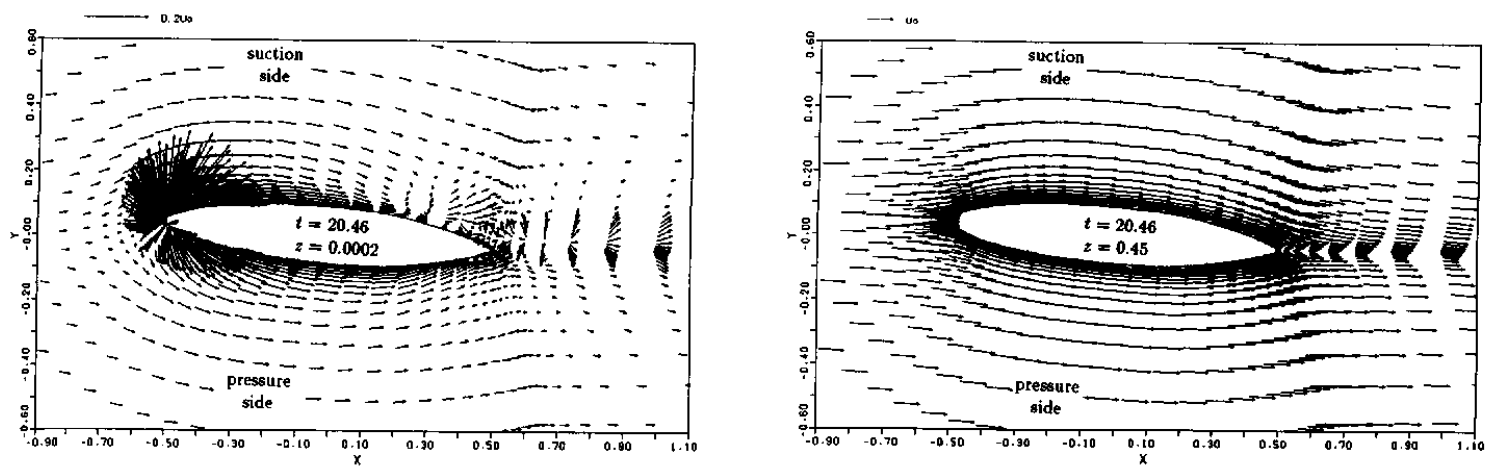

Fig. 14 Comparison of the Velocity Vectors between on the Plane of $z=0.0002$ and on the plane of $z=0.45: \alpha=-5^{\circ}$
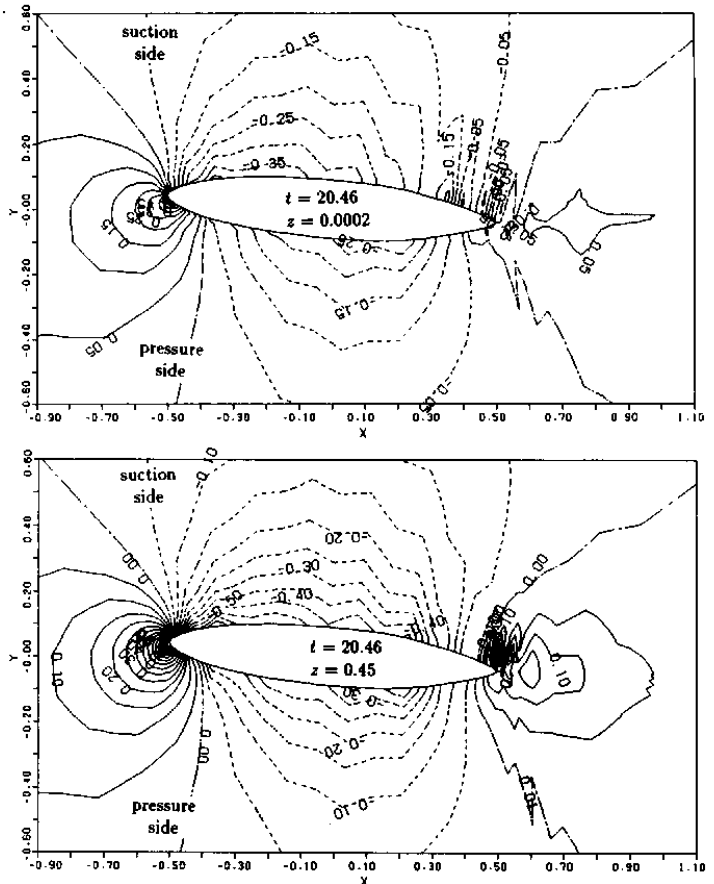

Fig. 15 Comparison of the Pressure between on the Plane of $z=0.0002$ and on the plane of $z=0.45$ : $\alpha=-5^{\circ}$

lift appears in Fig. 16. Near the plate, the pressure changes greatly with time near the trailing edge especially on the suction side. On the other hand, it does not change so much on the plane above the boundary layer. The mechanism of the unsteadiness for the flow is similar to that for the flow about the inclined strut. That is, the unsteady flow near the plate is due to the unsteady separation and attachment of the flow on the plate. In case of the inclined strut, the pressure oscillates largely on the $60^{\circ}$-side (see Fig. 10) where the flow

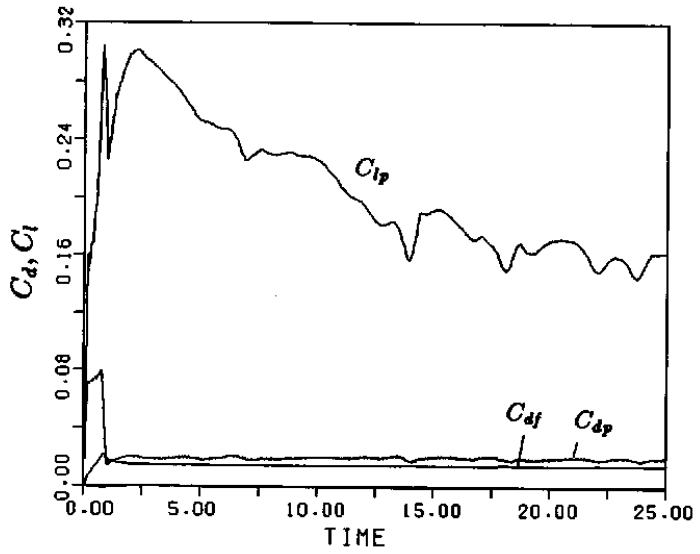

Fig. 16 Time History of the Forces Acting on the Strut : $\alpha=-5^{\circ}$

pattern changes largely with time. On the other hand, in case of the flow with an angle of attack, the flow pattern changes mainly on the suction side as can be expected from Fig. 14. so that the main change of the pressure is made on the suction side near the trailing edge as seen in Fig. 17. Note that the fluctuation of the pressure contributes to the increase of the lift for the flow for inclined strut but it does to the opposite for the flow with an angle of attack.

Fig. 18 shows the cross-flow velocity vectors and contours of the streamwise velocity at the corresponding times to those in Fig. 17; the section is the plane of $x=0.4$ on the suction side. They show quite different flows. At $t=21.96$, a new attachment point is seen around $y=0.06$ which makes the flow more complicated and seems to be the cause of the increase of the pressure on the suction side shown in Fig. 16. Such an unsteadiness of the flow is responsible for the fluctuation of the lift shown in Fig. 16, although further deep study must be necessary. 

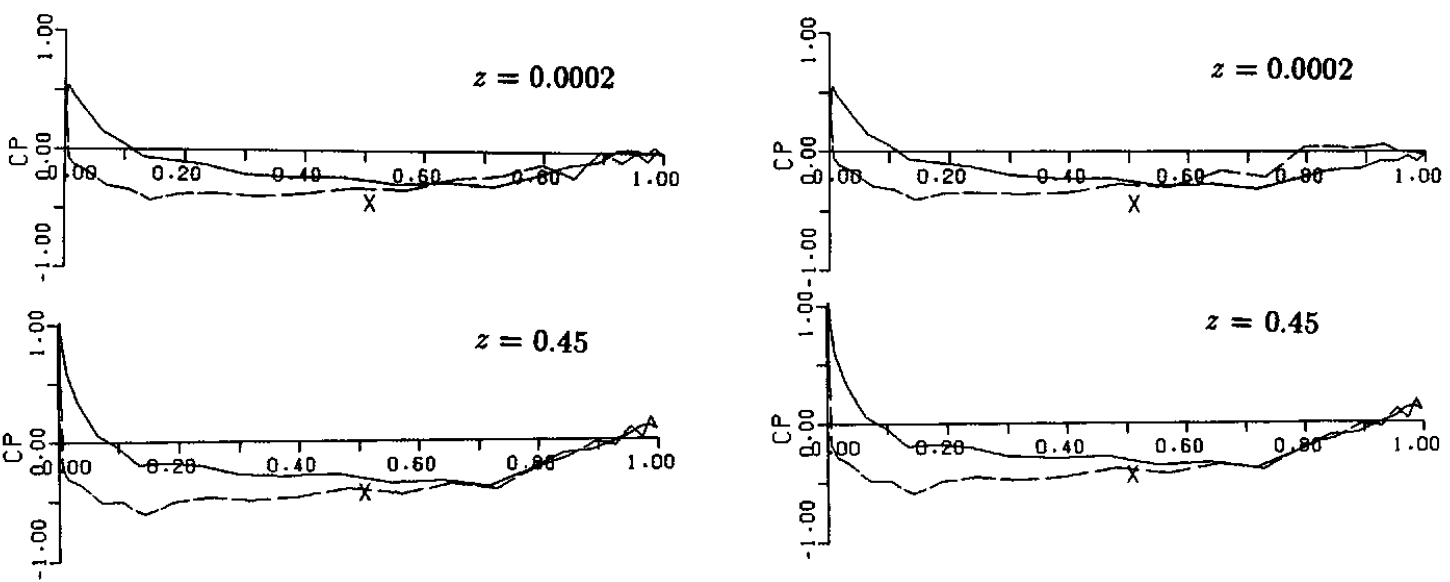

(a) $t=20.46$

(b) $t=21.96$

Fig. 17 Pressure Distribution along the Strut at Different Times; $\alpha=-5^{\circ}$
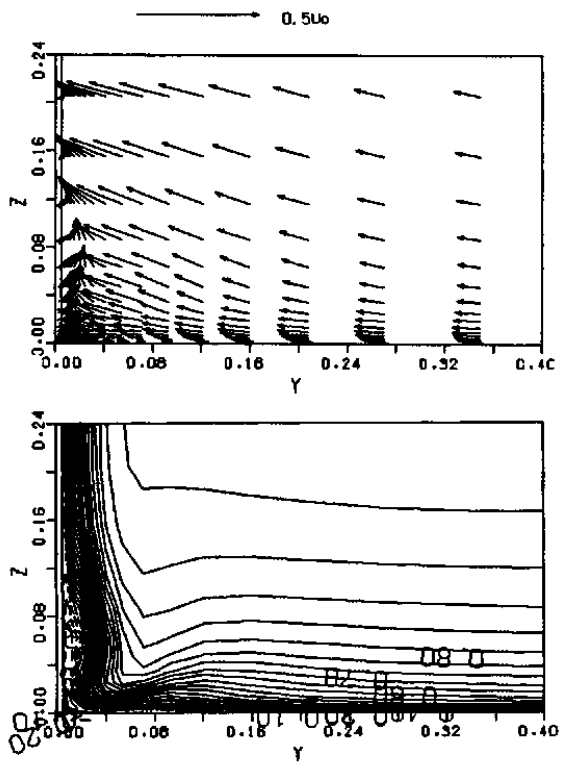

(a) $t=20.46$
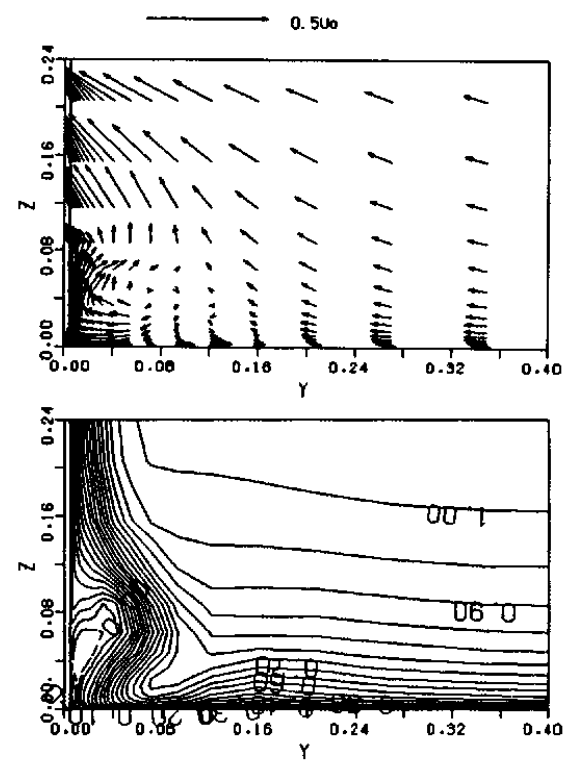

(b) $t=21.96$

Fig. 18 Cross Flow Velocity Vectors (above) and Contours of the Streamwise Velocity on the Plane of $x=0.4$ on the Suction Side : $\alpha=-5^{\circ}$

Fig. 19 shows the spanwise distribution of the sectional drag and lift coefficients of the strut. The computed force coefficients are obtained by averaging the data between $t=18.0$ and $t=24.0$. Frictional drag is similar to the case of the inclined strut while the pressure drag is rather small near the plate contrary to the inclined case. The lift reduces considerably near the plate which is due to the effects of plate. It should be also pointed out that appreciable effects of the plate up to the height far above the boundary layer.

\section{Conclusion}

Turbulent flow around the strut mounted on the plate is studied by solving Navier-Stokes equation. Turbu- 


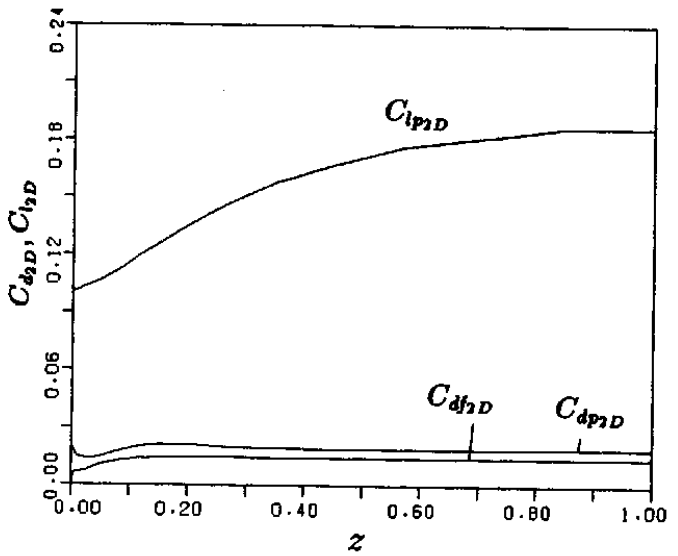

Fig. 19 Spanwise Distribution of the Sectional Drag and Lift Coefficients of the Strut : $\alpha=-5^{\circ}$

lence is simulated by Baldwin-Lomax model together with the Bleev's modified length scale which is introduced to treat the corner flow. The method is applied for two juncture flow simulations, one for the inclined strut and the other for the flow with an angle of attack.

At the Reynolds number of $10^{5}$, both flows show intensive unsteady behaviours near the plate while enough off the plate it is almost steady. It is seen that the main mechanism of the unsteadiness is due to the unsteady flows on the plate. The topology of the flow on the plate changes periodically which makes the forces acting on the strut oscillate.

It is found that the inclination of the strut induces a lift which is generated mainly near the plate. The measured pressure and forces also support this conclusion with which the computed results agree qualitatively well. The lift induced by the inclination of the strut by $30^{\circ}$ is almost equivalent to that by an angle of attack by $1^{\circ}$ for the strut with an one chord length of span.

On the other hand, the lift by an angle of attack reduces near the plate because of the low momentum of the oncoming boundary layer flow and insensitive change of the pressure along the strut near the plate.

The present computations are carried out with a relatively course grid. For further investigation of the flow characteristics, computations with finer grid are expected. Also, computations for higher Reynolds number flows would be interesting in the practical sense.

The authors wish to express their gratitude to Prof. Y. Doi at Hiroshima University for his discussion. They also wish to thank Mr. M. Takamatsu and Mr. H. Frui, graduate course students at Hiroshima University, who provide the experimental data referred in the present paper. All the computations were carried out using $\mathrm{H} /$ P720 workstation at Marine Hydrodynamics and Propulsion Laboratory of Hiroshima University.

\section{References}

1) Angui, J., Andreopoulos, J., : Experimental Investigation of a Three-Dimensional Boundary Layer Flow in the Vicinity of an Upright Wall Mounted Cylinder, J. of Fluid Engineering, Vol. 114, 1992.

2) Dickinson S. C., : An Experimental Investigation of Appendage-Flat Plate Junction Flow, DTN. SRDC Report DTNSRDC-86/052, 1986.

3) Pierce, F. J. and Shin, J., : The Development of a Turbulent Junction Vortex System, J. of Fluid Engineering, Vol. 144, 1992.

4) Devenport, W. J. and Simpson, R. L., : The Turbulence Flow Structure Near an AppendageBody Junction, 17th Symposium on Naval Hydrodynamics, 1988.

5) Chang, P.S. and Gessner, F. B., : Experimental Investigation of Flow About a Strut-Endwall Configuration, AIAA J., Vol. 29, No. 12, 1991.

6) Merati, P. and McMahon, H. M., : Experimental Investigation of a Turbulent Flow in the Vicinity of an Appendage Mounted on a Flat Plate, J. of Fluids Engineering, Vol. 113, 1991.

7) Sung, C. H. and Yang, C. -I., : Validation of Turbulent Horseshoe Vortex Flows, 17th Symposium on Naval Hydrodynamics, 1988.

8) Sung, C. H. and Griffin, M. J., : Improvements in Incompressible Turbulent Horseshoe Vortex Flow Calculations, AIAA Paper 91-0022, 1991.

9) Burke R. W., : Computation of Turbulent Incompressible Wing-Body Junction Flow, AIAA Paper 89-0279, 1989.

10) Chen, H.C., Patel, V.C., : The Flow Around Wing-Body Junctions, Proc. 4th Num. Physical Aspects Aerodynamic Flows, 1989.

11) Hoerner, S. F., : Fluid-Dynamic Drag, Published by the Author, 1965.

12) Kim, S. Y. and Mori, K., : A Study on Juncture Flows; Effects of the Inclination of Strut and the Curvature of Plate, J. of Soceity of Naval Architects of Japan, Vol. 172, 1992.

13) Briley, W. R. and McDonald, H., : Three-Dimensional Horseshoe Vortex Flow Using the Navier -Stokes Equations, 7th International Conference on Numerical Methods in Fluid Dynamics, Stanford University and NASA/Ames, 1980.

14) Kubendran, L. R., McMahon, H. M., Hubbartt, J. E., : Turbulent Flow around a Wing/FuselageType Juncture, AIAA J. Vol. 24, 1986.

15) Özcan, O., Ölcmen, M. S., : Measurement of Turbulent Flow Behind a Wind-Body Junction, Proceedings of the 6th Symposium on Turbulent Shear Flows, Toulouse, France, 1987.

16) Özcan, O., Ölcmen, M. S., : Measurement of Turbulent Flow Behind a Wing-Body Junction, AIAA J. Vol. 26, No. 4, 1988.

17) Wood, D. H., Westphal, R. V., : Measurement of the Flow Around a Lifiting-Wind/Body Junction, AIAA J. Vol. 30, No. 1, 1992.

18) Kim, S. Y. and Mori, K., : A Study on the Flow around a Strut Mounted on a Plate with an Angle of Attack, J. of Soceity of Naval Architects of Japan, Vol. 171, 1992.

19) Kim, S. Y., : A Study on the Flow around a Strut Mounted on the Plate, Ph. D. Thesis, Hiroshima 
University, 1993.

20) Baldwin, B.S. and Lomax, H., : Thin-Layer Approximation and Algebraic Model for Separatd Turbulent Flows, AIAA Paper 78-257, 1978.

21) Hung, C.M. and Buning, P. G., : Simulation of Blunt-Fin-Induced Shock-Wave and Turbulent Boundary-Layer Interaction, J. of Fluid Mechanics, Vol. 154, 1985.

22) Visbal, M. R. and Knight, D., : The BaldwinLomax Turbulence Model for Two-Dimensional Shock - Wave/Boundary - Layer Interactions, AIAA J., Vol. 22, No.7, 1984.
23) Hunt, J. C. R., Abell, C. J., Peterka, J. A. and Woo, H., : Kinematical Studies of th Flows around Free or Surface-Mounted Obstacles; Applying Topology to Flow Visualization, J. of Fluid Mechanics, Vol. 86, Pt. 1, 1978.

24) Takamatsu, M., Takebe, T., : Experimental Studies on Junction Flows, Graduate Thesis, Hiroshima Univ., 1992. (in Japanese)

25) Ooshita T., Furui, H., : Studies on Junction Flows: Measurement of Hydrodynamic Forces and Flow Visualization, Graduate Thesis, Hiroshima Univ., 1993. (in Japanese) 